\title{
From CVs to X-ray Binaries
}

\section{Jesús M. Corral-Santana*}

European Southern Observatory, Alonso de Córdova 3107, Casilla 19, Vitacura, Chile

E-mail: jcorraldeso.org

In the talk presented in GOLDEN2019, I wanted to show the similarities between binary systems containing white dwarf and those containing neutron stars or black holes. There are obvious differences due to e.g. the different strengths in the gravitational or magnetic field but this result is clearly biassed to show the properties that are more similar.

The Golden Age of Cataclysmic Variables and Related Objects V (GOLDEN2019)

2-7 September 2019

Palermo, Italy

${ }^{*}$ Speaker. 


\section{Introduction}

We all know that in a canonical model of an interacting binary, we have the primary (the compact object) which is accreting material coming from the secondary star, which, in turn, can be a giant, dwarf, red, blue, ... star that may be filling the Roche lobe. Thus, the primary is accreting via Roche lobe overflow through the inner Lagrangian point or, if the star has strong stellar winds, through them, and usually an accretion disc with a hot spot in the outskirts of the accretion disc.

If we consider the 3 types of compact objects, we can make a basic classification attending to different considerations (see also Figure 1):

- If we have a white dwarf (WD) as accretor, then we called them Symbiotics Stars if the secondary is a red giant and then, we divide them in Stellar-type (S-type) if the continuum spectrum of the giant star is not obscured or Dust type (D-type) if the spectrum is obscured.

- If the secondary star is a non-giant, then we called them Cataclysmic Variables (CVs) and we can divide them in non-magnetics (classical, recurrent, dwarf (DN) or nova-like), magnetic (polars, intermediate polars,...) or AMCVns which may not be considered as other CVs since they are formed by a pair of compact objects.

- If the compact object is either a neutron star (NS) or a black hole (BH), we called them Xray binaries and then we classify them according to the mass of the donor star in low-mass (LMXBs) or high-mass X-ray binaries (HMXBs). Even further, LMXBs are then divided in persistent or transients depending on the accretion rate and behaviour of the system, ...

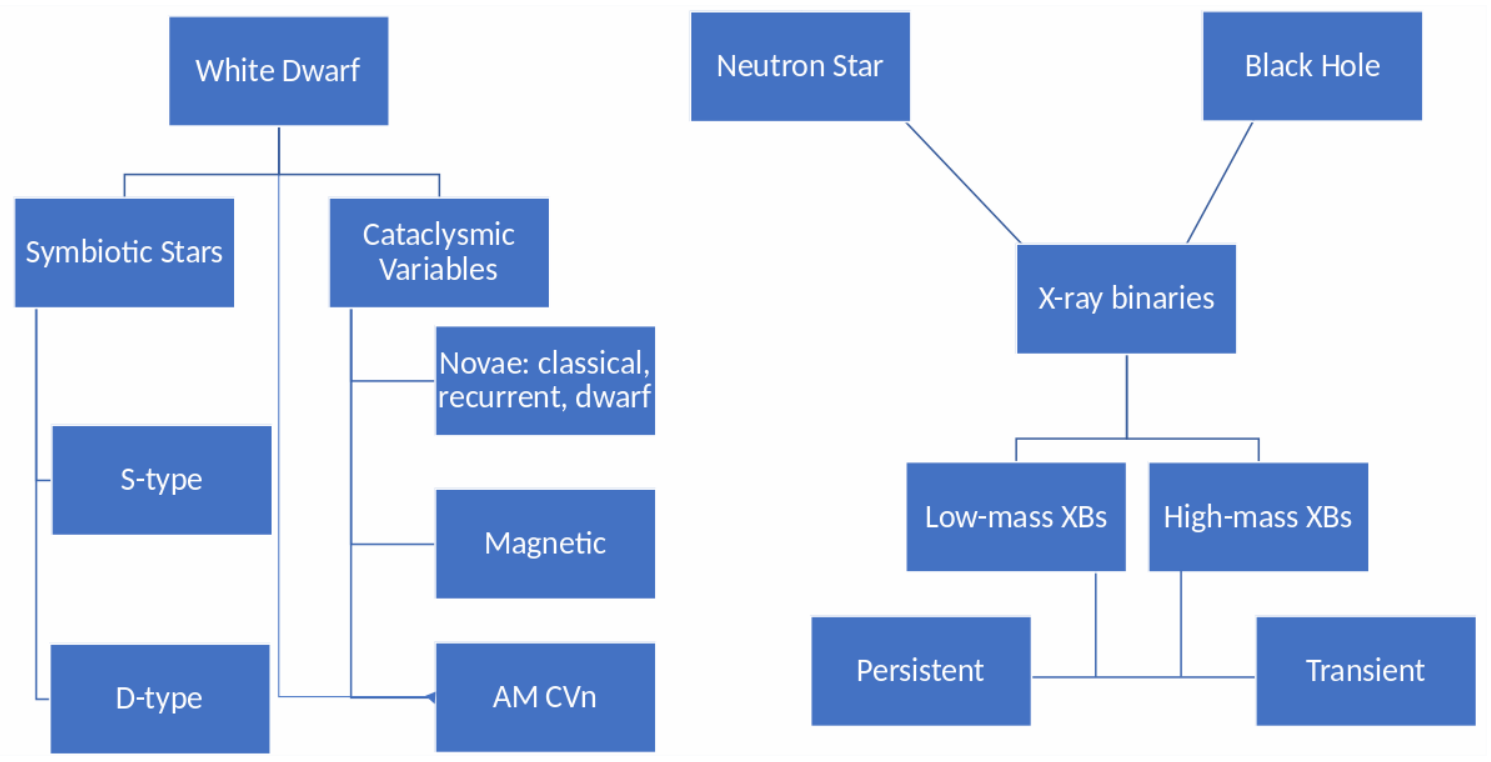

Figure 1: A basic concept of the zoo of interacting binaries.

To make it even more complicated, most of HMXBs contain NS but we only know 2 systems that have BHs. Also, most of persistent LMXBs sources harbour NS. Only transient LMXBs seem to be the systems where BHs are more easily found, however the number of detected systems is extremely low. So, why don't we look around and try to understand the whole picture? 


\section{Is there a single type of compact objects?}

There have been attempts to unify the whole zoo of interacting binary systems and even further. When I contacted Franco about the content of this talk, he pointed me out to some old works developed by [1] where he considered that all compact objects could be considered as a single type of gravimetric rotator, defined as any gravitationally-bounded object having an angular momentum and an intrinsic magnetic field, that may explain from AGNs or QSOs to NS pulsars or CVs. In this context and that time, the existence of BHs was not even confirmed undoubtedly and the existence of magnetic fields in stellar-mass BHs is still controversial.
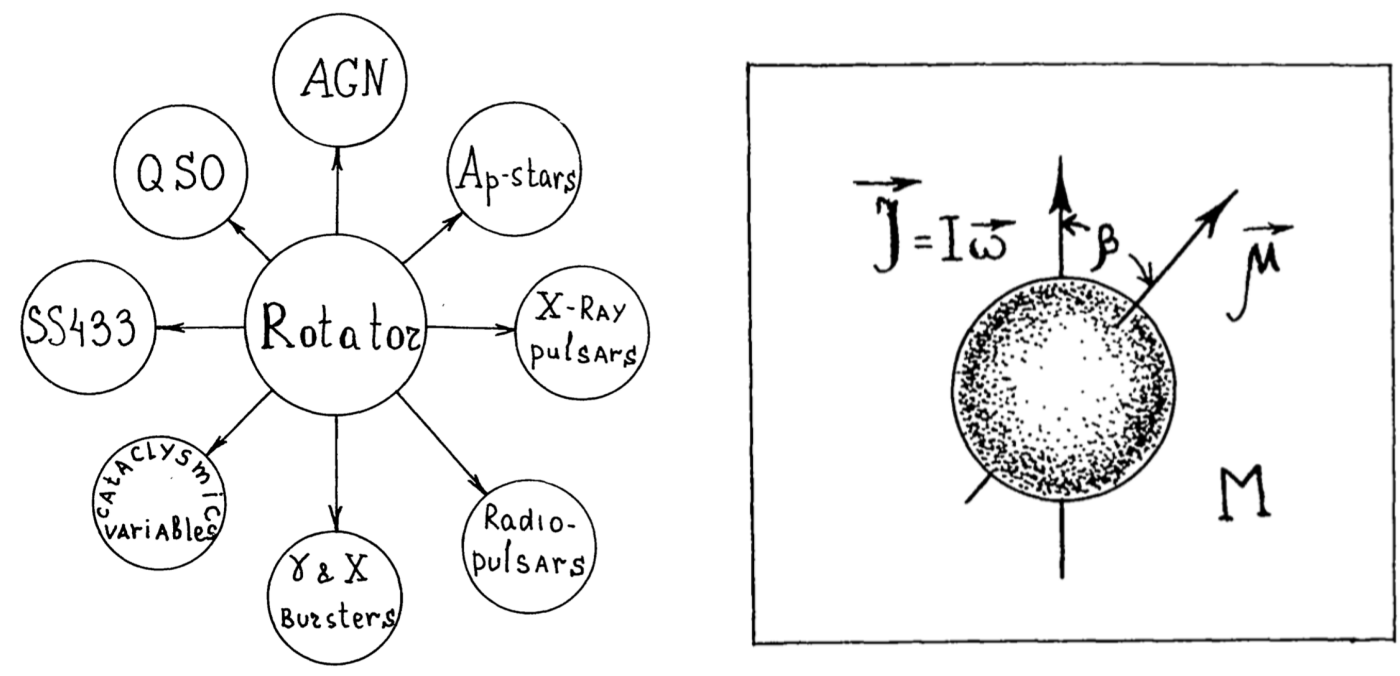

Figure 2: The concept of a gravimetric rotator introduce by Lipunov, defined by its angular momentum and magnetic field, not necessarily aligned.

More recently, [2] presented very interesting results trying to put together all the accretion mechanisms found in all sorts of sources. They started thinking: if stellar-mass BHs are scaled down version of Active Galactic Nuclei galaxies, can we extend this scale down to other systems regardless of the accretor? They found that the brightness variability of the accretion disc is similar, finding a linear correlation between the root mean square of the variability amplitude and the mean flux between AGNs, BHs, NS, WD and even Young Stellar Objects! When doing a Power Spectral Density (PDS), the power density gives the distribution of the variability as a function of frequency but there is always a break frequency in the slope of the PSD (see 3).

In the search of the dependences of this break frequency, they found that it is most sensitive to the radius of the accreting object rather than to the mass (see 4), and using information available of several types of accreting objects, they found this linear correlation between the break frequency predicted and observed. They concluded that the accretion-induced variability is universal across all types of accreting systems, compact or not.

If we focus on transient systems, basically LMXBs and probably Dwarf Novae, there are also similarities. Figure 5 shows the classical behaviour of transient XRBs in an intensity-color diagram in X-rays during the outburst [3]. The system goes into an outburst from quiescence, 


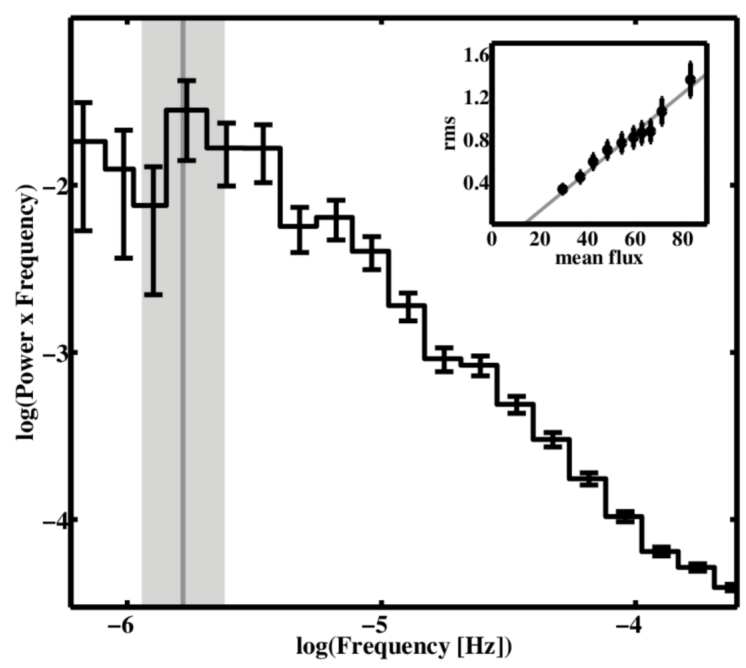

Figure 3: A power spectrum of the accreting young stellar object V866. Taken from [2]
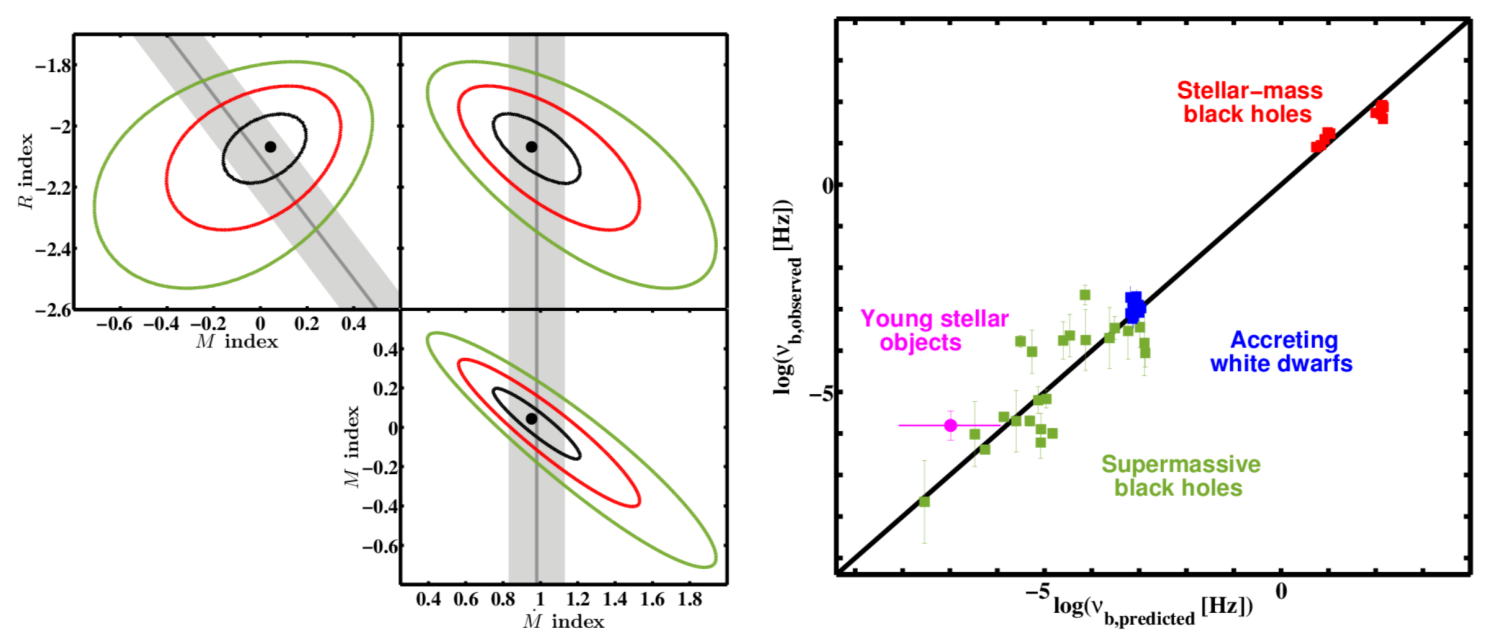

Figure 4: Taken from [2]. Left: Confidence contours for the mass, radius and mass accretion rate. Right: Edge-on projection of the accreting variability plane of a sample of young stellar objects, stellar-mass black holes, accreting white dwarfs and supermassive black holes.

reaching the low intensity - hard X-rays, transits to a softer state and decreases brightness towards the quiescence state.

[4] followed by [5] found a similar behavior in all 3 types of compact objects with a difference, in the case of WDs, it is not the X-ray hardness but the combination of the X-ray and Extreme UV luminosity.

What is key for BHs is that all systems detected so far have been found during outbursts. To date, we have not been able to find them while they are in quiescence as opposed to CVs, for instance, and this is our main bias. If we have a look at the current situation of $\mathrm{BH}$ transients (Figure 7), we have detected nearly 66 systems since 1966 but only 19 have been dynamically confirmed (including MAXIJ1820+070 recently confirmed [6]), which is a third of them. A few 


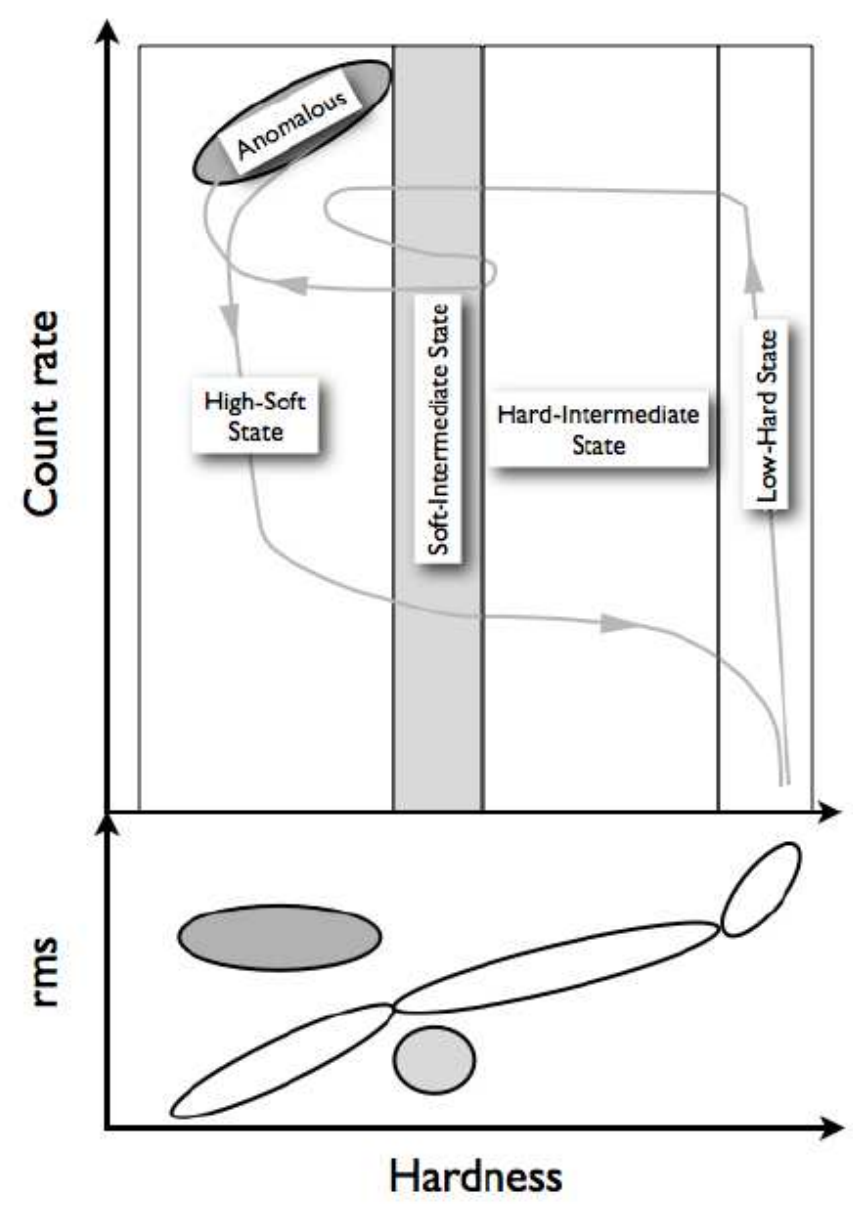

Figure 5: Hysteresis diagram (intensity versus hardness in X-rays) showed by BH transients during outburst, transiting between the different states. Taken from [3].

years ago we made some statistics focused on the observational information available about BHs and estimated a Galactic population of around 1300 BHTs [7]. Clearly, 19 are not sufficient to establish robust conclusions. The other main issue, as you know, is the faintness of the systems when they are back in quiescence and if we do not find the secondary, we cannot make dynamical studies, ...

Making a comparison between CVs and BHs we also find similar shapes in other properties like orbital periods. Of course, the sizes of the systems are different but this bimodal distribution of $\mathrm{P}_{\text {orb }}$ (Figure 8 right) resembles the one of CVs with the period gap (Figure 8 left), called as the bifurcation period, which is expected. It is interesting to note that some of the latest systems detected with BHs have less than $3 \mathrm{~h}$.

However, it is, in principle, not expected to have a mass gap between NS and BHs [7]. If we assume that the distribution of stellar masses is constant, there should not be a mass gap between 3 and $5 \mathrm{M}_{\odot}$. It is worth mentioning that we also have huge errorbars in the mass determination, in some cases up to $30 \%$ and very few robust mass determinations but still, this is not clear and it is still under debate. 


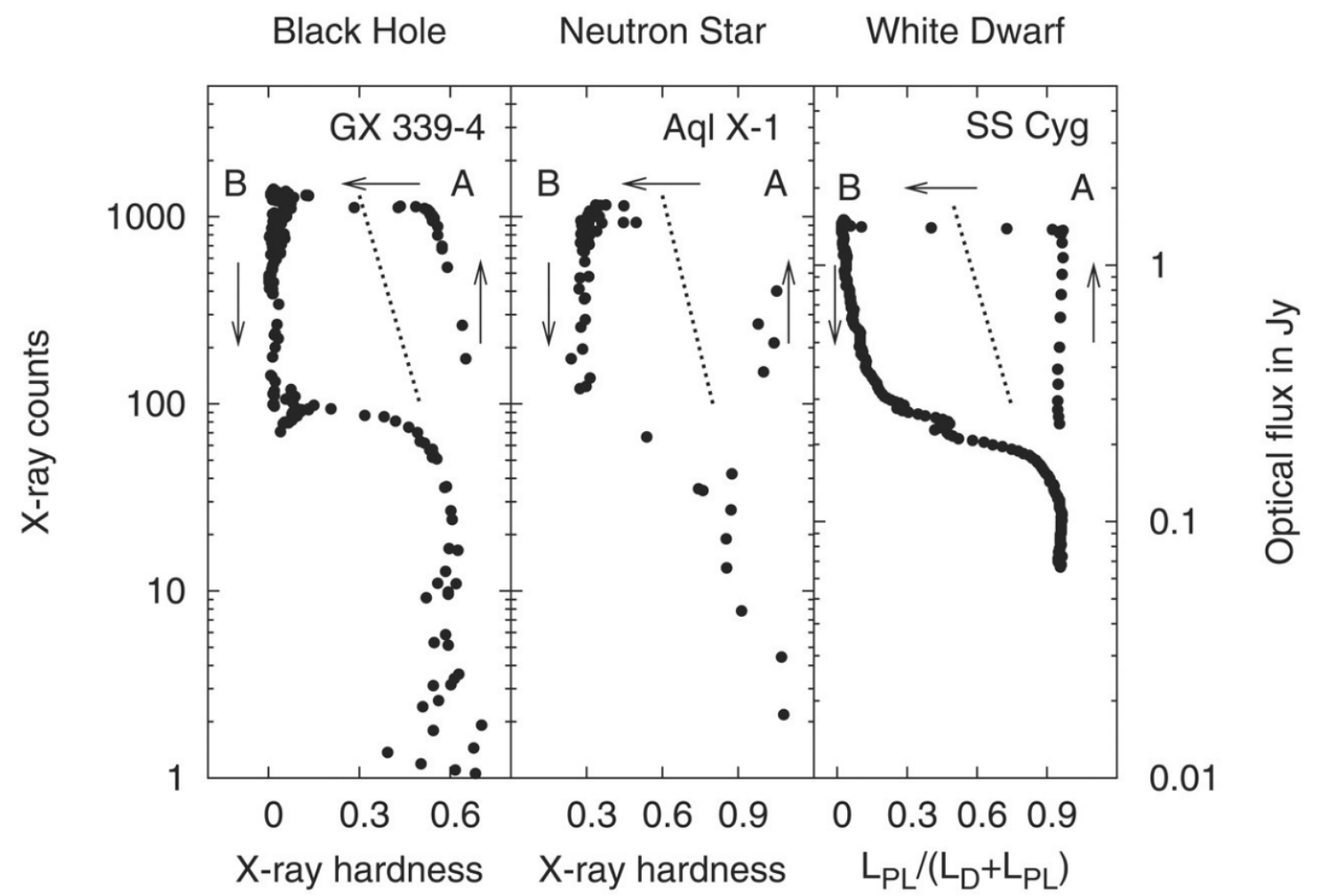

Figure 6: Hardness intensity diagram of a black hole, neutron star and white dwarf. Taken from [4] and updated by [5].

If we also take into account the BHs detected thanks to gravitational waves, we are still missing objects in this part of the mass distribution. Even the kilonova event was indicating this [8]. It seems that BHs discovered through microlensing events fill this gap [9] but the accuracy in their masses is less robust.

\section{Two interesting BHs challenging our understanding and opening new questions}

V404 Cyg was discovered in 1989 during an outburst event and later confirm as the first BH with a mass function larger than $6 \mathrm{M}_{\odot}$ [10]. In 2015, it had 2 new outbursts. In June 2015 it became the brightest $\mathrm{X}$-ray source in the sky and the brightest among the $\mathrm{BH}$ transients in the latest 10 years. There are a few things that were interesting about this outburst that are intriguing. The outburst lasted only 15 days, short compared to previous ones and other systems and finished abruptly but showing extremely fast variability [11]. Simultaneously to radio emission, they found a thermal cold wind moving at $1 \%$ of the speed of light, showed by a P-Cyg profile [12]. The presence of wind and jets is not easy to explain, before this outburst it was believed that this was not possible. Does this happen in CVs? What about other systems? [12] showed in a trailed spectrum taken on the 2nd day of the outburst, you can see the evolution of the P-Cyg emission with time. At the time of a huge reflare both in X-rays and optical, the wind disappears but immediately after it appears 


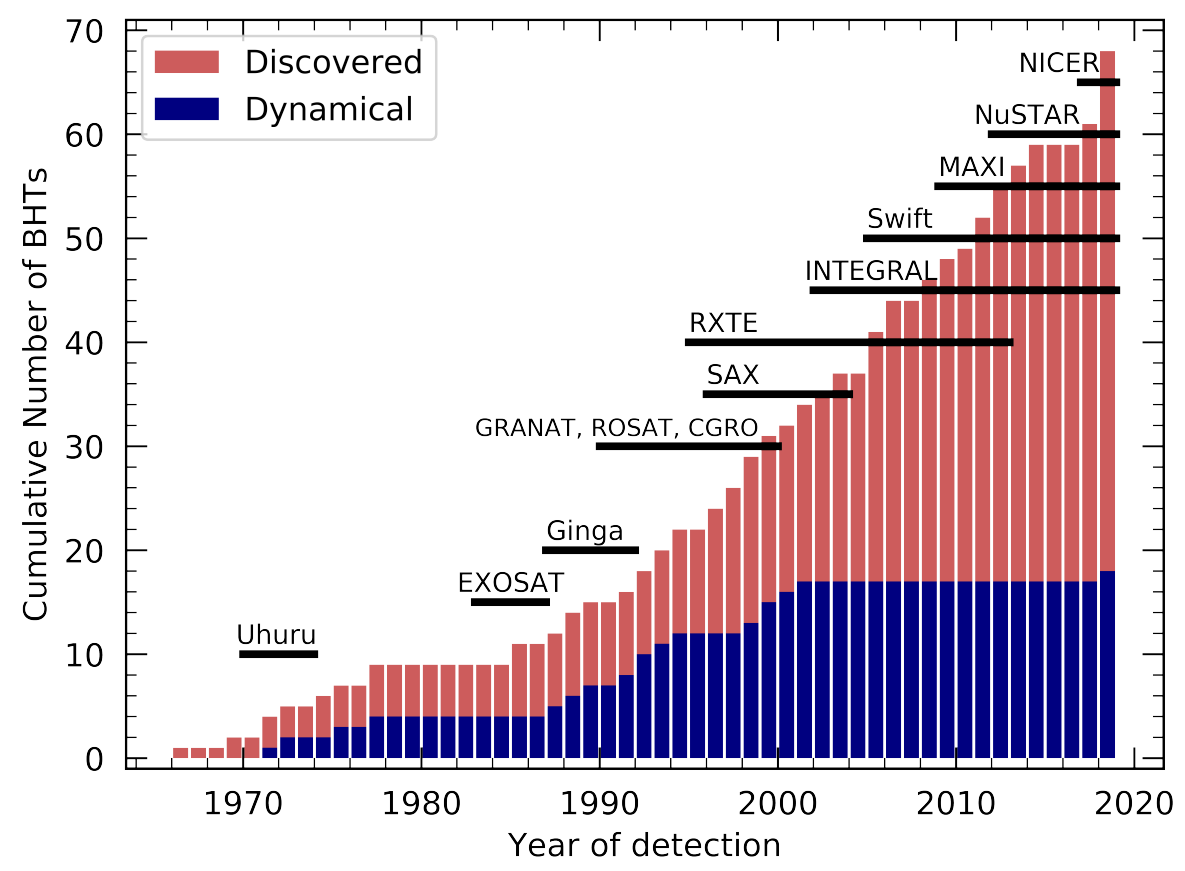

Figure 7: Cumulative histogram of the detected BH transients since 1960s (red) and dynamically confirmed BHs (blue). Taken from [7]
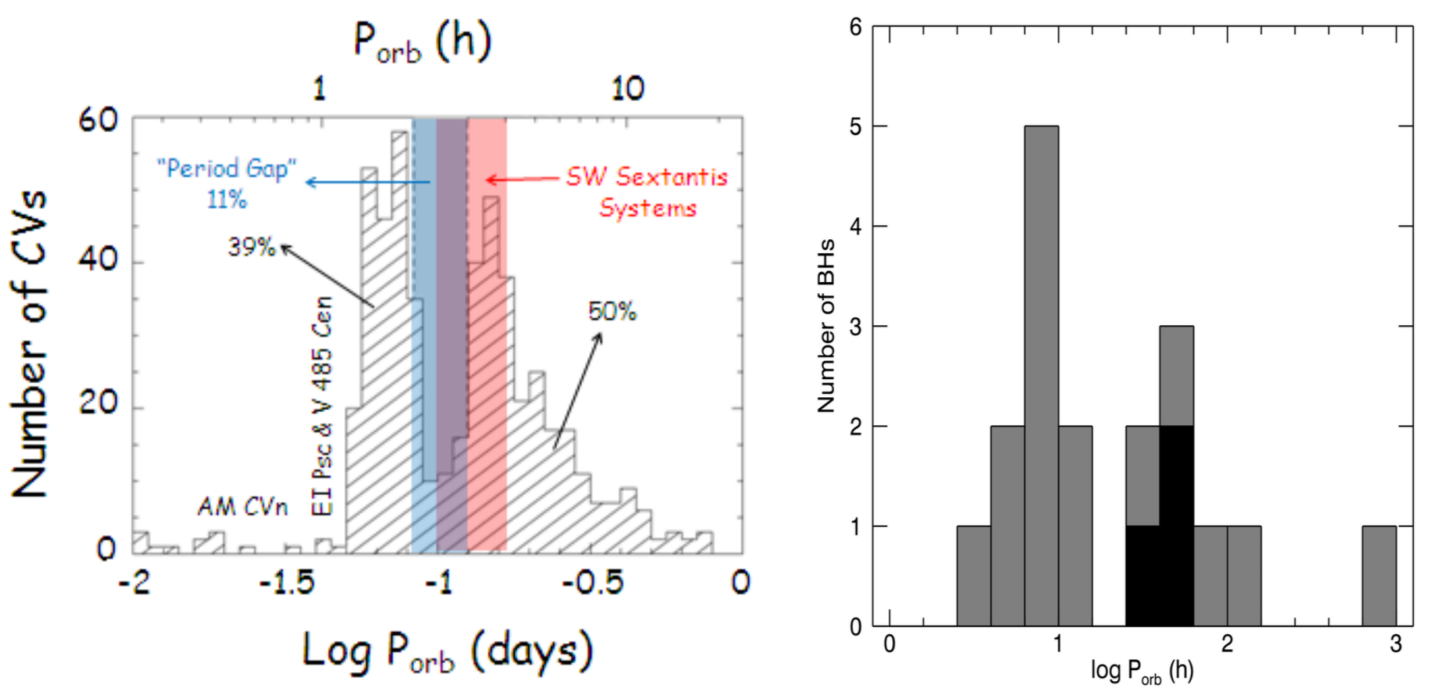

Figure 8: Left: period distribution of CVs (taken from [8]). Right: Period distribution of BH transients (taken from [7]). 

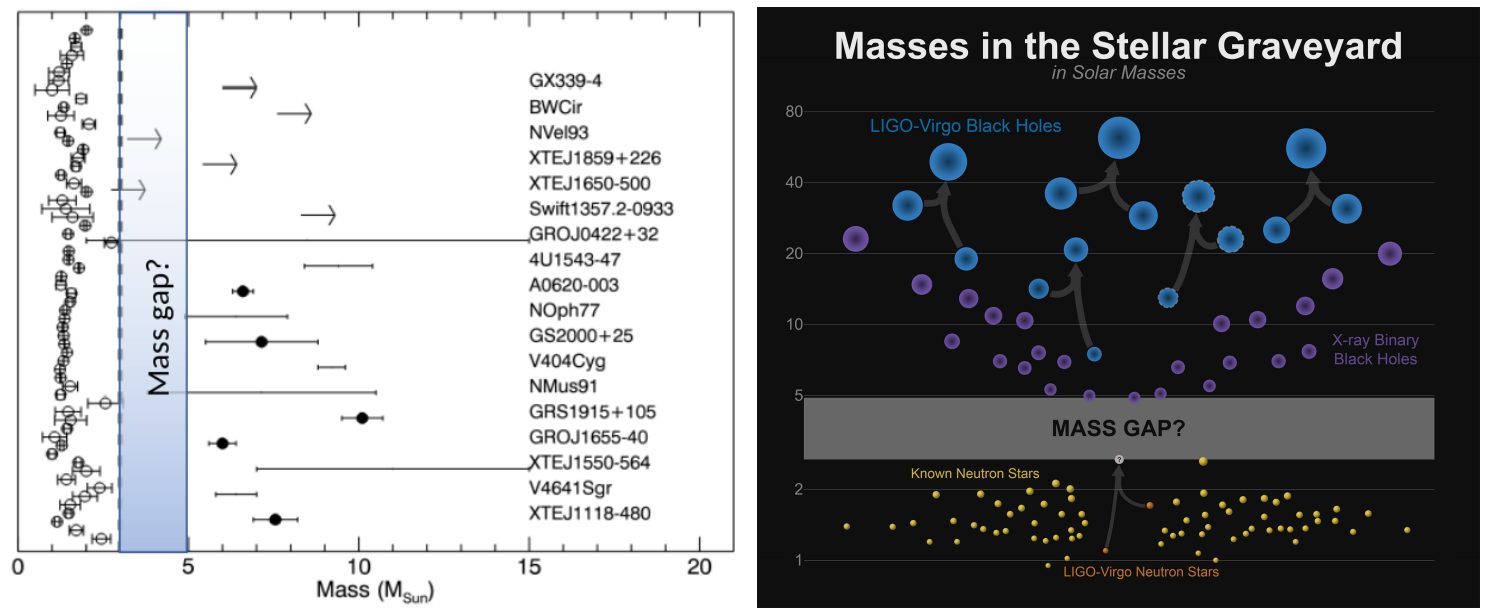

Figure 9: Left: Mass distribution of NS and BHs (updated from [7]). Right: Same as before but adding gravitational wave mergers [Image credit: LIGO-Virgo/Northwestern Univ./Frank Elavsky] .

stronger and both blue-shifter and red-shifted, what they interpret as an intense wind completely detached from the accretion disc. Also, at the end of the outburst, just a few days before it was abruptly finished and a nebular phase appeared: the emission lines start to become broader and brighter as you can see in this excerpt in 3 consecutive days [12].

Another system that opened new questions was Swift J1357.2-0933. This system went into outburst in 2011, we started to follow it up just immediately after it was detected and found extremely fast variability in the light curve with fast dips of up to $0.8 \mathrm{mag}$ or $50 \%$ of the flux in just $2 \mathrm{~min}$ (30\% of the flux in only 7s) [13]. Of course, the presence of dips implies a large inclination but we could not see any sign of eclipses. In addition, we noticed that the periodicity of the dips increased with days following the trend showed in Figure 10.

To explain this behaviour, we suggested the presence of a kind of wave or wall, clumpy and probably slightly tilted that was obscuring the reflected part of the inner accretion disc. This wave, probably caused by the outburst itself, was moving outwards in the accretion disc linked with the evolution of the outburst itself. We were able to measure the orbital period ( $2.8 \mathrm{~h})$ and radial velocity, yielding a mass function of more than $11 \mathrm{M}_{\odot}$. In 2017, it went into outburst again. We saw the same behaviour: very fast dips which recurrence period was increasing with time following almost the same behaviour than in 2011 (Figure 11; [14]).

Our colleagues in Southampton [15] also obtained very nice data completely independent from ours, obtaining the same results. Thanks to their multiwavelength coverage and timing, they updated the model including a jet and an extended corona, something that we commented in passing in our previous detection. Is this vertical structure ubiquitous in other accretion discs? Clearly, we managed to see it because is a system almost edge-on. Is this source special? If not, is this structure ubiquitous in all systems? 

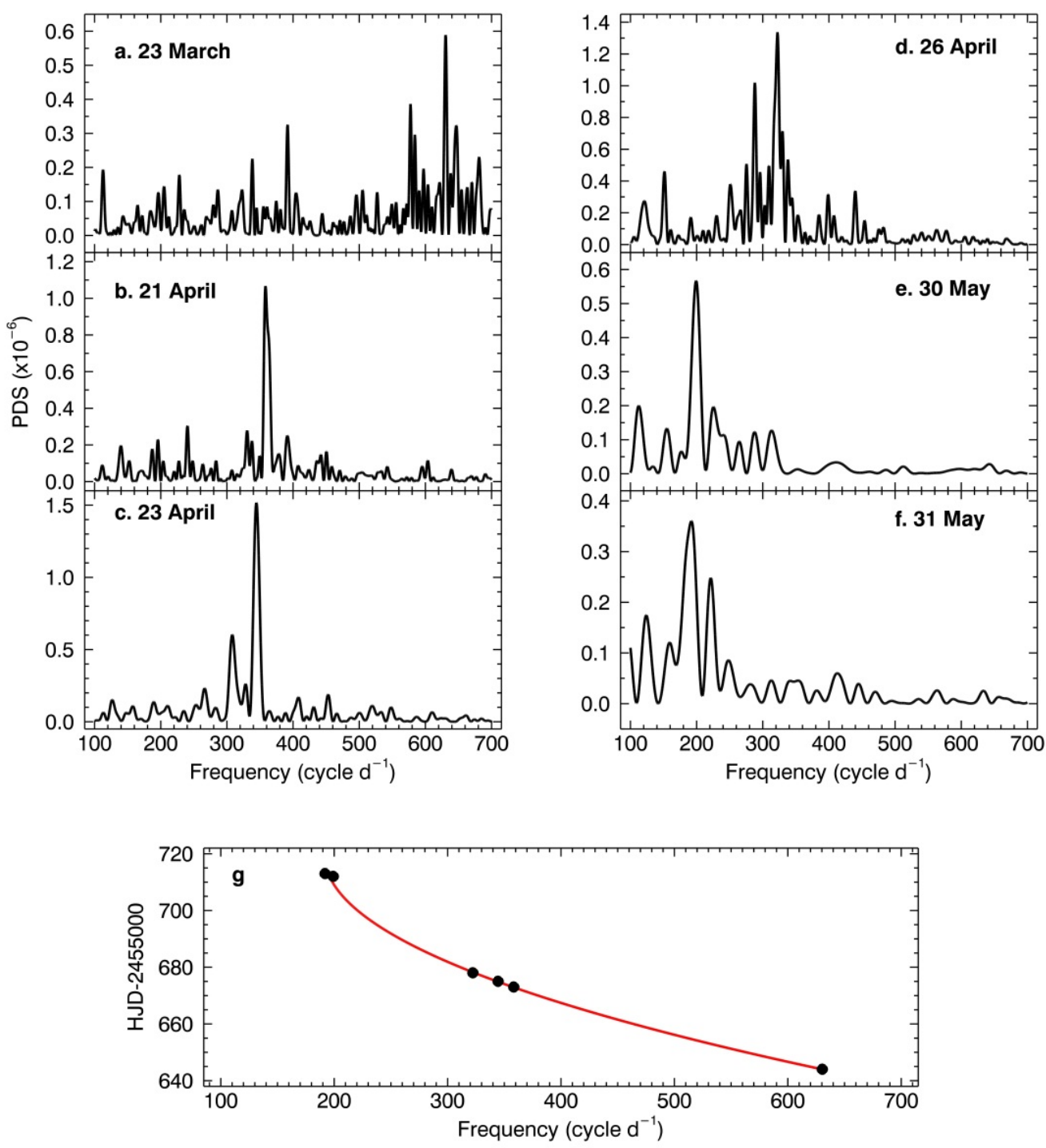

Figure 10: Periodogram showing the recurrence of the dips found in Swift J1357-0933 (a-f). Bottom: Change in the dip recurrence period. Taken from [13].

\section{Final questions}

But, among others, there are several open questions in the field:

- Is the mass gap real between NS and BHs? Is there a selection effect?

- Is there a cold wind present in all BHs?

- What about systems with other compact objects?

- Is there a vertical structure ubiquitous in all accretion discs?

- Increase the census and improve detection and confirmation of BH 

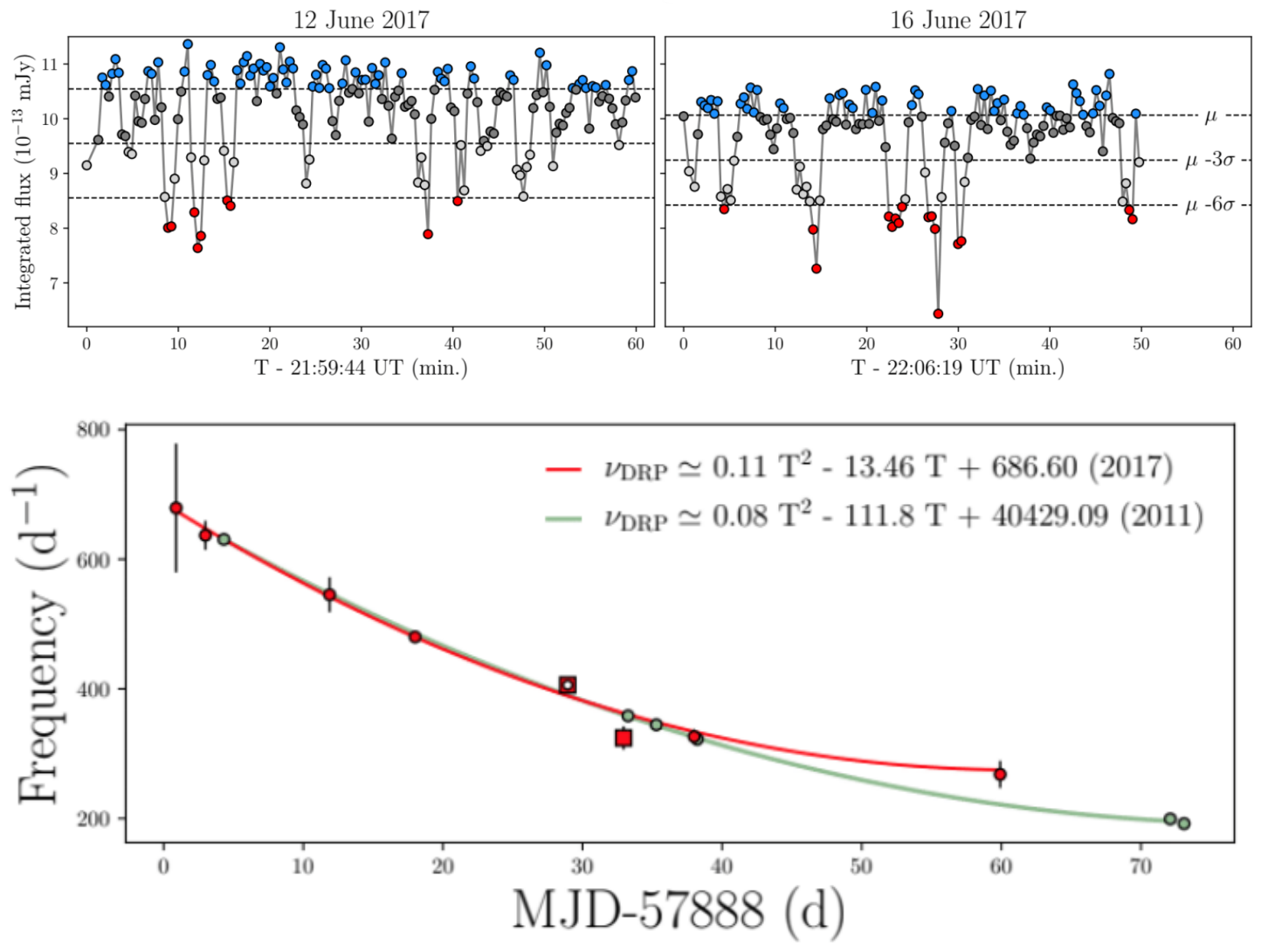

Figure 11: Fast variability found in Swift J1357-0933 during its 2017 outburst. Taken from [14].

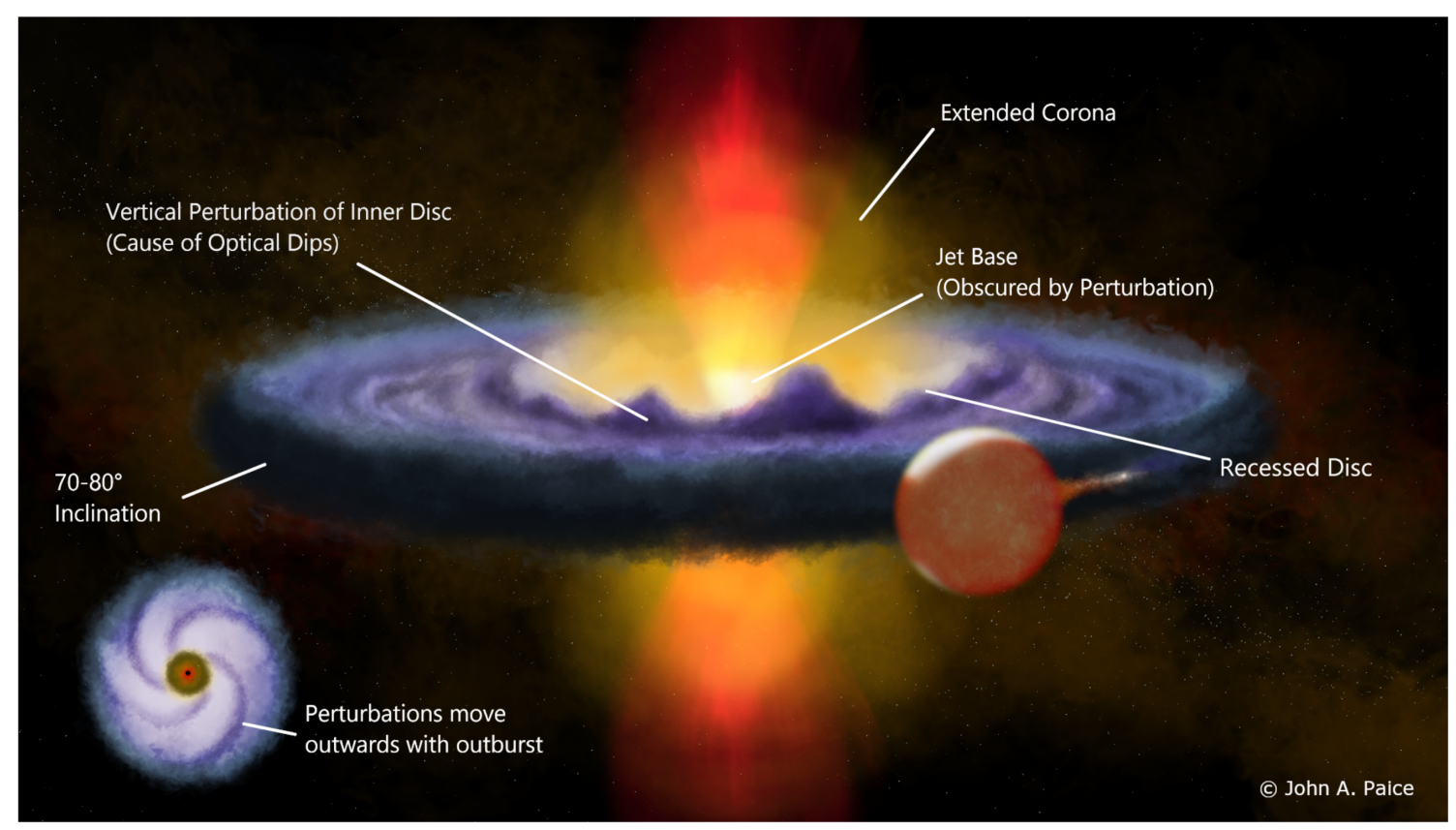

Figure 12: Updated model proposed by [15] of the system Swift J1357-0933 
- If accretion is universal, how can we learn from other systems:

To finish, I would like to end this proceeding paraphrasing Boris Gäensicke during a conversation in "La Gomera Accretion Week" workshop in 2017: How can we try to understand systems with BH in LMXBs, which are more difficult, if we do not fully understand CVs and learn from them?

\section{References}

[1] Lipunov, V. M. 1987. The Ecology of Rotators. Astrophysics and Space Science 132, 1.

[2] Scaringi, S., and 8 colleagues 2015 . Accretion-induced variability links young stellar objects, white dwarfs, and black holes. Science Advances 1, e1500686.

[3] Belloni, T. M., Motta, S. E., Muñoz-Darias, T. 2011. Black hole transients. Bulletin of the Astronomical Society of India 39, 409.

[4] Körding, E., Rupen, M., Knigge, C., et al. 2008, Science, 320, 1318

[5] Hameury, J.-M., Lasota, J.-P., Knigge, C., Körding, E. G. 2017. Hystereses in dwarf nova outbursts and low-mass X-ray binaries. Astronomy and Astrophysics 600, A95.

[6] Torres, M. A. P., and 6 colleagues 2019. Dynamical Confirmation of a Black Hole in MAXI J1820+070. The Astrophysical Journal 882, L21.

[7] Corral-Santana, J. M., Casares, J., Muñoz-Darias, T., Bauer, F. E., Martínez-Pais, I. G., Russell, D. M. 2016. BlackCAT: A catalogue of stellar-mass black holes in X-ray transients. Astronomy and Astrophysics 587, A61.

[8] Giovannelli, F., Sabau-Graziati, L. 2015. The Golden Age of Cataclysmic Variables and Related Objects: The State of Art.. The Golden Age of Cataclysmic Variables and Related Objects - III (golden2015) 1.

[9] Wyrzykowski, Ł., Mandel, I. 2019. Constraining the masses of microlensing black holes and the mass gap with Gaia DR2. arXiv e-prints arXiv:1904.07789.

[10] Casares, J., Charles, P. A., Naylor, T. 1992. A 6.5-day periodicity in the recurrent nova V404 Cygni implying the presence of a black hole. Nature 355, 614 .

[11] Gandhi, P., and 27 colleagues 2016. Furiously fast and red: sub-second optical flaring in V404 Cyg during the 2015 outburst peak. Monthly Notices of the Royal Astronomical Society 459, 554.

[12] Muñoz-Darias, T., and 9 colleagues 2016. Regulation of black-hole accretion by a disk wind during a violent outburst of V404 Cygni. Nature 534, 75.

[13] Corral-Santana, J. M., and 7 colleagues 2013. A Black Hole Nova Obscured by an Inner Disk Torus. Science 339, 1048.

[14] Jiménez-Ibarra, F., Muñoz-Darias, T., Casares, J., Armas Padilla, M., Corral-Santana, J. M. 2019. An equatorial outflow in the black hole optical dipper Swift J1357.2-0933. Monthly Notices of the Royal Astronomical Society 489, 3420.

[15] Paice, J. A., and 15 colleagues 2019. Puzzling blue dips in the black hole candidate Swift J1357.2 0933, from ULTRACAM, SALT, ATCA, Swift, and NuSTAR. Monthly Notices of the Royal Astronomical Society 488, 512. 
[16] Casares, J. 2016. Mass Ratio Determination from $\mathrm{H}_{\alpha}$ Lines in Black Hole X-Ray Transients. The Astrophysical Journal 822, 99.

[17] Zdziarski, A. A., Mikolajewska, J., Belczynski, K. 2013. Cyg X-3: a low-mass black hole or a neutron star.. Monthly Notices of the Royal Astronomical Society 429, L104.

[18] Belczynski, K., Bulik, T., Mandel, I., Sathyaprakash, B. S., Zdziarski, A. A., Mikołajewska, J. 2013. Cyg X-3: A Galactic Double Black Hole or Black-hole-Neutron-star Progenitor. The Astrophysical Journal 764, 96.

\section{DISCUSSION}

JOANNA MIKOLAJEWSKA's comment: Why Cyg X-3 is not included in the list? There is a paper published several years ago where we published a pretty robust mass determination suggesting that it contains a $\mathrm{BH}$ ?

JESUS CORRAL-SANTANA answer: First of all, because it has a Wolf-Rayet star companion and it is therefore a High-Mass X-ray binary (as Cyg X-1) but also because Cyg X-3 is a problematic source and we cannot rule out that it may harbour a NS. The paper you mention ([17]) suggest a mass for the compact object of $2.4_{-1.1}^{+2.1} \mathrm{M}_{\odot}$ which, in principle, would allow the existence of a $\mathrm{BH}$ within errors. It was followed by an interesting study about the fate of this binary as a possible BH-BH or BH-NS merger [18]. However, none of these publications rule out the possibility of a NS rather than a BH due to the extremely difficulty on isolating some parameters from the Wolf-Rayet companion.

MIKE SHARAS's Comment: Only a third ( $\approx 19$ of 66 ) BH X-ray systems have optical/IR counterparts. Why? What is being done to improve this?

JESUS CORRAL-SANTANA answer: There are a few more systems with optical/IR counterparts detected, there are also some with counterparts detected even in quiescence. However, it is true that only around a third of the whole sample of BH candidates have dynamical confirmation of a BH. The main issue in the dynamical confirmation is obtaining the radial velocity of the secondary. It requires not only the detection of the counterpart but also obtaining time-resolved spectroscopy covering, at least, the orbital period with good signa-to-noise ratio. With current instrumentation, this is limited to systems with $R \lesssim 22$. With future $30-40 \mathrm{~m}$ telescopes we will be able to measure it in fainter systems. In addition, a new empirical relation between the full-width at half-maximum of the $\mathrm{H} \alpha$ line and the radial velocity of the secondary star has was recently proposed [16]. This powerful technique will allow us to constrain the radial velocity just with one spectrum, and therefore estimate the mass of the compact object.

MAREK ABRAMOWICZ's Comment: At such a large inclination, general relativistic effects in light propagation are known to be large. Have you included them by ray tracing? 
JESUS CORRAL-SANTANA answer: No, we haven't, but this is an interesting point that we should follow up. Thanks a lot!

LINDA SCHMIDTOBREICK's Comment: If you add the WD in the mass distribution, would you get another gap between WDs and NSs? Especially for binaries, WD mass are rather low due to common envelope evolution, so the formation scenario would be responsible for the maximal reachable mass within a type

JESUS CORRAL-SANTANA answer: It doesn't seem so. If we have a look at Figure 9, we can see that there are NS with masses in all ranges from 1.4 to $2.2 \mathrm{M}_{\odot}$ (e.g. MSP J0740+6620), suggesting that the mass distribution is continuous. Actually, there are NSs with masses slightly below the Chandrasekhar limit (e.g. PSR J0737-3039B with $1.25 \mathrm{M}_{\odot}$ ).

DAVID BUCKLEY's Comment: Our recent SALT spectroscopy of Swift J1357-0933 during the recent outburst in May/June 2019 shows transient absorption lins (Balmer and He II) which are blue-shifted, but decelerate to rest velocity over the timescale of the dip $(\sim 200 \mathrm{~s}$.

JESUS CORRAL-SANTANA answer: This is an amazing result. Thanks for letting me know about it. I look forward to seeing those data!

NOEL CASTRO SEGURA's Comment: Do we see an extended coronal emission in AWD as the one seen in the BHT Swift J1357.2-0933?

JESUS CORRAL-SANTANA answer: Yes, this was suggested in WZ Sge and Simone Scaringi et al. research. 